\title{
Comparação do Desempenho dos Fundos de Ações Ativos e Passivos
}

\author{
Bruno Ribeiro Castro* \\ Andrea Maria Accioly Fonseca Minardi**
}

Resumo

O objetivo deste trabalho é investigar se os gestores de fundos de ações ativos tiveram habilidade superior de seletividade em relação ao equilíbrio de mercado e aos fundos passivos no Brasil. Para isso, foram analisadas séries mensais de retornos líquidos e de estimativas de retornos brutos de 626 fundos de ações, no período entre janeiro de 1996 e outubro de 2006. A metodologia adotada foi a análise do sinal e significância da constante alfa da regressão dos retornos em excesso dos fundos em relação aos fatores propostos por Carhart (1997) com a adição de um fator de market timing. Encontraram-se evidências de que um número significativo de fundos de ações ativos explora anomalias conhecidas, como tamanho, razão entre valor contábil e valor de mercado do patrimônio líquido, momento e market timing. Os resultados indicam que apenas 4,8\% dos fundos ativos apresentou alfa significativamente positivo quando se analisa retornos líquidos, e em média os alfas dos fundos ativos não são significativamente diferentes dos fundos passivos. Quando são considerados os retornos brutos, o número sobe para 10,3\%, e os alfas dos fundos ativos são em média positivos. Essa evidência está de acordo com a versão de eficiência de mercado de Jensen (1978), segundo a qual o preço dos ativos reflete as informações existentes até o ponto em que os benefícios marginais do uso das informações (lucros a serem realizados) não excedem os custos marginais.

Palavras-chave: seleção de ativos; desempenho de fundos de ações; fundos ativos; eficiência de mercado.

Códigos JEL: G11; G14.

\section{Abstract}

We intend to investigate whether active portfolio managers have higher security selection ability than passive managers in Brazil. We built net monthly historical returns and estimated gross historical returns series from January 1996 till October 2006 of 626 stock mutual funds. We used the regression model proposed by Carhart (1997) with the addition of a market timing factor and analyzed the alpha coefficient sign and significance. Our results show that a significant number of managers exploit well-known strategies as size, book-to-market ratio, momentum and market timing. When we use net returns series as the dependent variable, we find that only $4.8 \%$ of active portfolios have positive and significant alphas. Active

Submetido em Outubro de 2008. Aceito em Abril de 2009. O artigo foi avaliado segundo o processo de duplo anonimato além se de ser avaliado pelo editor. Editor responsável: Ricardo P. C. Leal.

* Insper Instituto de Ensino e Pesquisa. E-mail: brunorcastro@gmail.com

** Insper Instituto de Ensino e Pesquisa. E-mail: minardi@isp.edu.br 
portfolio performance on average is not significantly different than passive portfolio performance. But when we run the regressions using the estimated gross returns, we found that $10.3 \%$ of active funds have positive and significant alphas, and on average the performance of active funds is significantly positive. Our results are in accordance with Jensen's (1978) version of efficient market, in which asset prices reflect existing information till the moment when marginal benefits of using information do not exceed marginal costs.

Keywords: security selection; stock mutual fund performance; active portfolio management; market efficiency.

\section{Introdução}

A gestão ativa dos fundos, com base em análise fundamentalista, procura selecionar títulos mal apreçados, com o intuito de obter um retorno superior ao de equilíbrio de mercado. A gestão passiva procura replicar um índice de mercado, objetivando o retorno de equilíbrio. De acordo com a Hipótese de Eficiência de Mercado na forma semi-forte, os preços dos ativos refletem todas as informações publicamente disponíveis. Dessa forma, a análise fundamentalista não seria útil para encontrar constantemente títulos mal apreçados, e conseqüentemente num mercado eficiente os fundos ativos não conseguiriam obter retornos ajustados ao risco superiores aos de equilíbrio de mercado e aos fundos passivos. No período de janeiro de 1996 a outubro de 2006, o mercado de fundos de ações no Brasil apresentou um forte crescimento. Segundo os dados analisados da ANBID (Sistema de Informações ANBID versão 4.1), o número de fundos de ações era de apenas 175 em janeiro de 1996 e passou para 512 em outubro de 2006. Dos fundos analisados nesse trabalho, 92,17\% eram de gestão ativa, e o volume aplicado em gestão ativa foi quase 17 vezes superior ao aplicado em fundos passivos. Esses dados sinalizam que tanto os investidores quanto a indústria de fundos acreditam que existem ineficiências de mercado a serem exploradas.

O objetivo deste artigo é testar se a estratégia ativa de seletividade tem obtido retorno superior à estratégia passiva no longo prazo, justificando a maior procura dos investidores brasileiros por fundos ativos de ações. Para isso, foram coletados dados mensais de 626 fundos de investimento em ações, sendo 577 ativos e 49 passivos no período de janeiro de 1996 a outubro de 2006. Foram analisadas tanto rentabilidades líquidas, apuradas pelas cotas divulgadas do fundo, como estimativas de rentabilidades brutas, acrescentando-se aos retornos líquidos o percentual cobrado de taxa de administração divulgado pelos fundos, porém sem levar em conta taxas de performance e custos de corretagem. Como os fundos ativos cobram tarifas superiores às dos fundos passivos, é possível que mesmo que os gestores tenham gerado retorno anormal por habilidade superior para explorar ineficiências, o investidor não se beneficie desse ganho superior, por este ser diluído no pagamento das taxas de administração, desempenho e outros custos de transação. 
A metodologia de teste consistiu em observar o sinal e a significância das constantes alfas das regressões corrigidas pelo procedimento de Newey-West dos retornos em excesso de cada um dos fundos em relação aos fatores sugeridos por Carhart (1997) e por um fator de market timing.

As regressões realizadas com retornos líquidos indicam que foram muitos pou$\cos$ os fundos ativos que apresentaram habilidade de seletividade significativa, $\mathrm{e}$ que em média os alfas das regressões foram negativos e significativamente diferente de zero. Não houve evidências de que em média os fundos ativos tiveram desempenho superior aos fundos passivos.

Outra característica identificada foi o significativo número de fundos ativos que exploram fatores de características conhecidos como tamanho, razão entre valor contábil e de mercado do patrimônio líquido, momento e market timing. Identificou-se também que quanto maior o fundo, melhor o seu desempenho para o investidor.

Quando se analisam retornos brutos, observa-se um maior número de fundos com desempenho superior ao equilíbrio e um número menor de fundos com alfa negativo, ou seja, que destruiu valor. Nota-se também uma diminuição da concentração dos piores desempenhos em fundos pequenos quando se utiliza retornos brutos. Este fato indica que possivelmente a taxa de administração elevada não permite que o investidor tire proveito das habilidades superiores dos gestores.

Apesar dos resultados mais favoráveis à criação de valor com a análise com retornos brutos, observa-se apenas $10,3 \%$ de fundos com desempenho superior ao equilíbrio. Esse percentual ainda é pequeno, sugerindo que os ativos de renda variável no mercado brasileiro estão em média bem apreçados.

O restante do artigo está organizado como segue. A Seção 2 contém uma revisão de literatura sobre desempenho de fundos em mercados internacionais e brasileiro; a Seção 3 explica a base de dados e a metodologia utilizada no artigo; a Seção 4 apresenta e discute os resultados e por fim, a Seção 5 conclui o artigo.

\section{Revisão da Literatura}

Fama (1972) classifica as estratégias de gestão ativa em dois tipos: seletividade e market timing. A seletividade mensura o quão bem evolui a carteira escolhida comparada a um índice de referência com o mesmo nível de risco. Nada mais é do que uma análise micro, na qual se busca prever o movimento dos preços das ações, e assim comprar as que podem dar maiores retornos no futuro e vender as que podem dar piores retornos. Já o market timing é uma análise macro, na qual se procura prever ciclos econômicos e medir o retorno da parcela da carteira exposta ao risco. Mediante esse procedimento o gestor pode alterar as quantias alocadas em ativos com risco e ativo livre de risco.

São vários os trabalhos que investigam se os gestores de fundos são bem sucedidos em estratégias de seletividade e market timing e se existe persistência de desempenho. Não existe, porém, um consenso sobre habilidade superior de fundos ativos. 
Jensen (1968) adota o Capital Asset Pricing Model (CAPM) como modelo de equilíbrio e analisa a significância e o sinal das constantes alfas das regressões do excesso de retorno dos fundos em relação ao excesso de retorno de índices de mercado. Um alfa significativamente positivo é uma evidência de que o gestor foi hábil em seletividade, um alfa não significativamente diferente de zero indica que não há evidências de habilidade superior de seletividade e um alfa significativamente negativo indica que o desempenho foi inferior ao do equilíbrio de mercado. Jensen analisou retornos anuais de 115 fundos no período de 1945 a 1964 e encontrou poucos casos de alfas positivos e significativos, concluindo que não há evidências de habilidade superior de seletividade.

Henriksson (1984) investiga habilidade superior de seletividade e market timing. Analisa retornos mensais de 116 fundos do mercado norte americano no período de fevereiro de 1968 a julho de 1980, dividindo em dois sub-períodos e usando as técnicas paramétricas e não paramétricas desenvolvidas em Henriksson e Merton (1981). Apenas um dos fundos apresentou coeficientes significantes e positivos de market timing e de seletividade em ambos sub-períodos. Lee e Rahman (1990) analisaram a habilidade de seletividade e market timing em 93 fundos entre janeiro de 1977 e março de 1984. Corrigindo as regressões por heteroscedasticidade, 14 dos fundos apresentaram habilidade positiva e significante de seletividade e 16 dos fundos em market timing.

Grinblatt e Titman (1989) defendem que taxa de administração e de performance e outros custos de transação acabam por diluir ou exaurir ganhos anormais dos gestores provenientes de habilidades superiores. Constroem séries de tempo de retornos líquidos e brutos de uma amostra grande de fundos mútuos com base nas carteiras declaradas nos relatórios trimestrais. Para estimar a série de retornos brutos consideram despesas de administração, corretagens e outros custos de transação. Com base no alfa obtido com o modelo de Jensen (1968) aplicado a estimativas de retornos brutos, encontram evidências de retornos superiores, especialmente para fundos de crescimento. A evidência não é encontrada com retornos líquidos. Eles completam afirmando que os fundos de crescimento incorrem em maiores custos de transação e taxas, diluindo os ganhos anormais.

Hendricks et alii (1993) analisaram dados trimestrais de 165 fundos mútuos norte-americanos no período entre 1974 e 1988. Encontraram evidências de que existe o fenômeno de hot hands, ou seja, de que os fundos que tiveram o melhor desempenho relativo continuam a ter o melhor desempenho no próximo trimestre, mas que esse é predominante um fenômeno de curto prazo, com pico em torno de um ano. Também encontraram o fenômeno inverso, o icy hands, ou seja, de que os fundos que tiveram o pior desempenho relativo continuam a ter o pior desempenho relativo também no horizonte de até um ano. A magnitude do efeito de icy hands é superior à do efeito de hot hands, e embora existam poucas evidências de que os melhores desempenhos sejam sustentáveis no longo prazo, foram encontradas algumas evidências de persistência de piores desempenhos no longo prazo. 
Fama e French (1993) discutem que a carteira de mercado não engloba todos os riscos sistemáticos, e que, portanto, o CAPM não poderia ser considerado um modelo adequado de equilíbrio de mercado. Por isso sugerem que além da carteira de mercado sejam incluídos fatores de risco capturados pelo efeito tamanho e razão entre o valor contábil e o valor de mercado do patrimônio líquido.

Carhart (1997) amplia a discussão adicionando ao modelo de três fatores de Fama e French o fator de momento de um ano. Com seu modelo de quatro fatores conclui não haver evidências da existência de Alfas positivos significativos, indicando a inexistência de retornos anormais por parte da gestão ativa. Ele ainda aconselha os investidores a evitar fundos com baixo desempenho e diz que fundos com retornos elevados em um ano tendem a obter retornos superiores no ano seguinte, porém o mesmo não ocorre nos anos subseqüentes. Por fim, afirma que os custos de transação têm efeitos negativos diretos no desempenho dos fundos, mostrando que elevadas taxas de administração prejudicam a rentabilidade.

No mercado brasileiro os resultados dos diferentes trabalhos também não mostram um consenso quanto à existência de evidências de desempenho superior dos fundos. Andaku e Pinto (2003) encontraram evidências de persistência de longo prazo no desempenho dos fundos de ações no período de julho de 1994 até junho de 2001. Monteiro (2007) encontra evidência de persistência de desempenho em períodos de 3 meses a um ano, tanto na habilidade de seletividade como em market timing.

Franz e Figueiredo (2003) examinaram 454 fundos de ações entre o período de dezembro de 1994 a dezembro de 2000 e não encontraram evidências de habilidade de market timing.Leusin e Brito (2006) encontraram evidências de retornos superiores em alguns poucos fundos de gestão ativa, provenientes de estratégia de seletividade e market timing. O período analisado foi de 1998 a 2003.

Rochman e Eid Junior (2006) analisaram os desempenhos de 699 fundos ativos e passivos, divididos em fundos de ações, cambiais, multimercados e renda fixa, no período entre janeiro de 2001 e janeiro de 2006. Encontraram evidências de habilidade superior para os fundos de multimercado e fundos de ações, mas não em fundos de renda fixa, onde se percebeu muitos casos de alfas significativamente negativos.

\section{Metodologia}

\subsection{Base de dados}

Os dados de cotas de fundo, foram obtidos do sistema de Informações da ANBID (SI-ANBID) versão 4.1. Foram coletadas cotas de fechamento mensais de fundos de ações classificados pela ANBID como abertos no período entre janeiro de 1996 a outubro de 2006. Não foram incluídos na base de dados fundos classificados como fechados, de ações setoriais, de capital protegido, de capital estrangeiro, de privatização, de investimento imobiliário, offshore, de previdência, FICs e FACs. A amostra final resultou em 626 fundos de 293 instituições diferen- 
tes. Dentre os gestores incluem-se bancos de investimentos, bancos comerciais e instituições independentes.

Os retornos mensais dos fundos foram estimados por retornos simples, de acordo com a equação (1).

$$
R_{i, t}=\left(P_{i, t}-P_{i, t-1}\right) / P_{i, t-1}
$$

onde $R_{i, t}$ é o retorno do fundo $i$ no mês $t, P_{i, t}$ o valor da cota do fundo $i$ no mês $t$ e $P_{i, t-1}$ o valor da cota no mês $t-1$.

A Tabela 1 mostra a quantidade de fundos por modalidade de gestão e benchmark, a Tabela 2 a quantidade de fundos por faixa de patrimônio líquido (apurado no último mês em que se observou cota para o fundo) e a Tabela 3 apresenta estatísticas descritivas do patrimônio líquido dos fundos.

Tabela 1

Perfil da amostra para análise de retornos líquidos em termos de modalidade de gestão e benchmark

\begin{tabular}{|c|c|c|c|c|}
\hline \multirow[t]{2}{*}{ Benchmark } & \multirow[t]{2}{*}{ Fundos } & \multicolumn{2}{|c|}{ Modalidade } & \multirow{2}{*}{$\begin{array}{c}\text { Fundos } \\
\text { Alavancados }\end{array}$} \\
\hline & & Ativos & Passivos & \\
\hline IBOVESPA & 524 & 484 & 40 & 124 \\
\hline IBX & 91 & 82 & 9 & 7 \\
\hline IBA & 11 & 11 & 0 & 0 \\
\hline Total & 626 & 577 & 49 & 131 \\
\hline
\end{tabular}

Informação (SI-ANBID) versão 4.1.

Tabela 2

Quantidade de fundos por faixa de patrimônio líquido

\begin{tabular}{l|c}
\hline \multicolumn{1}{c|}{ Patrimônio } & Qtde Fundos \\
\hline Até 1 MM & 81 \\
Entre 1 MM e 5 MM & 129 \\
Entre 5 MM e 10MM & 72 \\
Entre 10MM e 20MM & 73 \\
Entre 20MM e 30MM & 69 \\
Entre 30MM e 40MM & 41 \\
Entre 40MM e 50MM & 28 \\
Entre 50 MM e 100MM & 58 \\
Entre 100 MM e 500MM & 69 \\
Entre 500 MM e 1BM & 6 \\
\hline Fonte: Análise dos autores, com base nos da- \\
dos do Sistema de Informação (SI-ANBID) \\
versão 4.1.
\end{tabular}


Tabela 3

Dados descritivos de patrimônio líquido por tipo de gestão (em R \$ 000)

\begin{tabular}{l|c|c|c}
\hline & Total & Ativos & Passivos \\
\hline Médio & 46.877 .929 & 47.932 .582 & 34.178 .152 \\
Máximo & 1.655 .389 .375 & 1.655 .389 .375 & 244.322 .512 \\
Mínimo & 12.278 & 12.278 & 429.616 \\
Desvio Padrão & 110.994 .914 & 114.580 .814 & 49.730 .893 \\
\hline Total & 29.345 .583 .512 & 27.705 .032 .220 & 1.640 .551 .292 \\
\hline Fonte: Análise dos autores, com base nos dados do Sistema de Informação \\
(SI-ANBID) versão 4.1.
\end{tabular}

Na Tabela 1, observa-se que $83 \%$ dos fundos têm como referência o IBOVESPA, e que $92 \%$ dos fundos da base de dados são de gestão ativa. Dos 626 fundos, 131 são alavancados, ou seja, admitem uma exposição ao risco superior ao patrimônio do fundo.

A classificação dos fundos não foi constante ao longo do período analisado. Isso é devido à mudança de regulamentação da CVM : instrução 302 em 1999 e instrução 409 em 2004 e devido a vontade dos administradores. Neste artigo considerou-se que a última classificação permaneceu constante ao longo de todo o período analisado. Essa simplificação não causou grandes impactos, pois os principais objetos de análise: tipo de gestão (ativa e passiva) e benchmark permaneceram inalterados durante o período analisado. Os fundos classificados como "ações outros" foram analisados tendo como benchmark o IBOVESPA.

Na Tabela 2 observa-se que $20 \%$ da amostra total encontra-se na faixa de patrimônio líquido entre $R \$ 1$ milhão e $R \$ 5$ milhões, e mais da metade da amostra concentra-se na faixa de $\mathrm{R} \$ 1$ milhão a 30 milhões. Considerou-se para essa análise o patrimônio líquido no mês em que se observou a última cota do fundo.

$\mathrm{Na}$ Tabela 3 nota-se que existe uma grande diversidade de tamanho de fundos, sendo o patrimônio líquido do menor de $\mathrm{R} \$ 12$ mil e do maior superior a $\mathrm{R} \$$ 1,65 bilhões. Os fundos passivos são em média menores que os fundos ativos, e os tamanhos dos fundos apresentam dispersão menos acentuada. O volume total investido nos fundos ativos é quase 17 vezes superior ao investido em fundos passivos.

Utilizou-se a taxa SELIC over como proxy para o retorno do ativo livre de risco $\left(R_{f}\right)$, pois acredita-se que este é o custo de oportunidade de um ativo livre de risco para um investidor de um fundo de ação. Apesar da caderneta de poupança apresentar menor volatilidade, espera-se que um investidor que aplique seus recursos em fundos de ações tenha a mesma facilidade, conhecimento e informação para aplicar seus recursos em ativos atrelados à SELIC.

Utilizaram-se o IBOVESPA, IBX e IBA como índices de mercado. Os retornos dos índices $\left(R_{m i}\right)$ foram estimados de acordo com a equação (1), e utilizou-se os preço médio dos índices no último dia de cada mês, uma vez que as cotas de fundos de ações são apuradas com os preços médios das ações no dia. Esses valores, bem como os retornos da SELIC over foram coletados da base de dados da Economática. 
Os fundos incluídos na amostra tiveram uma vida em média de cinco anos e dois meses, com um desvio-padrão de 2,6 anos. A moda de sobrevivência dos fundos observada foi de dois anos, enquanto que o período mínimo que um fundo durou foi 16 meses. Por outro lado, alguns fundos persistiram por mais de 10 anos. A Tabela 4 contém uma análise da variação do número de fundos em cada um dos anos analisados. Dos 203 fundos existentes ao longo de 1996, ano quando se começou a coletar os dados, 144 foram liquidados, ou seja, apenas 59 fundos existiram durante todo o período analisado. Dos 626 fundos analisados, 376 foram liquidados antes do encerramento do período analisado. Controlou-se os efeitos de fundos que trocaram de nome, foram incorporados ou eliminados por não terem alcançado desempenho satisfatório para não gerar viés de sobrevivência.

Tabela 4

Análise da entrada e liquidação de fundos na amostra durante o período analisado

\begin{tabular}{l|ccccccccccc}
\hline \multicolumn{1}{c}{ Fundos } & \multicolumn{11}{c}{ Ano } \\
\cline { 2 - 11 } & 1996 & 1997 & 1998 & 1999 & 2000 & 2001 & 2002 & 2003 & 2004 & 2005 & 2006 \\
\hline $\mathrm{N}^{0}$ entrantes & 203 & 141 & 52 & 38 & 36 & 59 & 35 & 32 & 30 & 0 & 0 \\
$\mathrm{~N}^{\mathrm{o}}$ liquidações & 0 & 0 & 25 & 41 & 36 & 57 & 39 & 63 & 51 & 49 & 15 \\
total & 203 & 344 & 371 & 368 & 368 & 370 & 366 & 335 & 314 & 265 & 250 \\
\hline
\end{tabular}

Fonte: Análise dos autores, com base nos dados do Sistema de Informação (SI-ANBID) versão 4.1.

Adotou-se que a taxa de administração dos fundos no último mês de cota observada persistiu durante toda a sua existência. Essa simplificação foi feita por não ter sido possível obter os dados de todos os meses de taxa de administração. Quando a ANBID não informou a taxa de administração, essas foram coletadas do site da CVM e do programa Quantum. Entretanto, mesmo assim, não foi possível levantar taxa de administração para todos os fundos, o que restringiu a amostra de fundos com rentabilidade bruta para 507. Dentre estes 507 fundos, 17 apresentaram taxa de administração igual zero, fazendo com que os resultados obtidos com as regressões brutas fossem iguais aos obtidos com as rentabilidades líquidas.

Foi considerada que a taxa divulgada por ANBID, CVM e Quantum é a efetivamente cobrada dos investidores, embora diversas vezes esse não seja o caso, pois para alguns fundos a taxa de administração é variável, e depende da estratégia utilizada e ganho auferido. Não foram estimadas taxa de performance, pois não há dados sobre ela no sistema SI_ANBID. Também não foi possível estimar custos de corretagem, por não se conhecer com os dados desse trabalho as carteiras dos fundos e o rebalanceamento destas.

A Tabela 5 contém o perfil da amostra de fundos para a análise de retornos brutos em termos de benchmark e modalidade de gestão. As Tabelas 6 e 7 contêm dados descritivos da taxa de administração. Pode-se perceber que em média os fundos ativos cobram tarifas superiores às dos fundos passivos, conforme era esperado, dado que incorrem em maiores gastos. O máximo de tarifa cobrada por um fundo ativo foi $12 \%$ e do fundo passivo $6 \%$. Também existe uma maior dispersão na tarifa cobrada dos fundos ativos em relação aos passivos. 
Tabela 5

Perfil da amostra para análise de retornos brutos em termos de modalidade de gestão e benchmark

\begin{tabular}{l|c|c|c|c}
\hline Benchmark & Fundos & \multicolumn{2}{|c|}{ Modalidade } & \multirow{2}{*}{$\begin{array}{c}\text { Fundos } \\
\text { Allavancados }\end{array}$} \\
\cline { 3 - 4 } & & Ativos & Passivos & 113 \\
\hline IBOVESPA & 425 & 384 & 41 & 7 \\
IBX & 80 & 73 & 7 & 0 \\
IBA & 2 & 2 & 0 & 120 \\
\hline Total & 507 & 459 & 48 & \multicolumn{3}{l}{}
\end{tabular}

Informação (SI-ANBID) versão 4.1

Tabela 6

Quantidade de fundos por faixa de taxa de administração

\begin{tabular}{l|c}
\hline \multicolumn{1}{c}{ Taxa } & Número de Fundos \\
\hline $0 \%-1 \%$ & 191 \\
$1 \%-2 \%$ & 101 \\
$2 \%-3 \%$ & 78 \\
$3 \%-4 \%$ & 96 \\
Acima de 5\% & 41 \\
\hline \multicolumn{2}{l}{ Fonte: Análise dos autores, com base nos da- } \\
dos do Sistema de Informação (SI-ANBID) \\
versão 4.1.
\end{tabular}

Tabela 7

Dados descritivos de taxa de administração por modalidade de gestão e benchmark

\begin{tabular}{l|c|c|c|c|c}
\hline & Ativos & Passivos & IBOVESPA & IBX & IBA \\
\hline Média & 2,03 & 1,51 & 2,07 & 1,41 & 1,85 \\
Máximo & 12,00 & 6,00 & 12,00 & 6,00 & 3,00 \\
Mínimo & 0,00 & 0,00 & 0,00 & 0,00 & 0,00 \\
Desvio Padrão & 1,93 & 1,70 & 1,96 & 1,59 & 1,63 \\
\hline \multicolumn{7}{l}{ Fonte: Análise dos autores, com base nos dados do Sistema de }
\end{tabular}

Informação (SI-ANBID) versão 4.1 .

\subsection{Metodologia adotada}

Para testar a existência de habilidade superior de seletividade dos fundos de ações ativos foram feitas regressões múltiplas do retorno em excesso de cada um dos fundos em relação aos 4 fatores de Carhart (1997) e foi adicionado um fator de market timing. Foram construídas séries históricas mensais dos fatores de Carhart para o período de janeiro de 1996 a outubro de 2006.

Carhart (1997) sugere quatro fatores de risco sistemáticos: (1) o excesso de retorno de um índice de ações $\left(R m_{i}-R_{f}\right)$; (2) uma carteira auto-financiada comprada nas ações de menor tamanho e vendida nas ações de maior tamanho (SMB); (3) uma carteira auto-financiada comprada nas ações de maior razão entre valor contábil e valor de mercado do patrimônio líquido e vendida nas ações de menor razão (HML); (4) uma carteira auto-financiada comprada nas ações vencedoras (maiores retornos realizados) e vendida nas perdedoras (piores retornos realizados) - (WML).

Os índices IBOVESPA, IBX e IBA foram utilizados como proxy da carteira de mercado. Quando o benchmark do fundo era o IBOVESPA, a variável de excesso 
de retorno da carteira de mercado representa a diferença entre os retornos do IBOVESPA e do ativo livre de risco, e de maneira análoga para os fundos indexados ao IBX e ao IBA.

Para a construção das séries históricas dos fatores SMB (efeito tamanho), HML (razão entre valor contábil e de mercado do patrimônio líquido) e WML (momento), foram coletados na Economática os preços de fechamento de todas as ações brasileiras existentes negociadas na BOVESPA, o valor de mercado do patrimônio líquido de cada uma dessas empresas e o valor patrimonial da ação no período entre julho de 1995 e outubro de 2006. Calculou-se a razão entre valor contábil e valor de mercado do patrimônio líquido da ação i no mês t como sendo a razão entre o valor patrimonial da ação $i$ no mês $t$ dividido pelo preço da ação $i$ no mês $t$. Os retornos das ações foram estimados de acordo com a equação (1).

Para construir as séries de retornos do fator SMB, as ações foram ordenadas mensalmente em ordem crescente de acordo com o valor de mercado do patrimônio líquido (tamanho). As ações sem cotação foram eliminadas da amostra, resultando numa média de 291 ações aproveitadas em cada um dos meses. As ações foram alocadas mensalmente em cinco carteiras, sendo a primeira carteira composta pelas menores ações (S) e a quinta carteira a de maiores ações (B). Calculou-se o retorno mensal de cada carteira como sendo a média simples das ações que compunham essa carteira naquele mês. A série mensal de retorno do fator SMB foi estimada pela diferença do retorno mensal da carteira 1 (S) e da carteira 5 (B). As séries de retornos do fator HML e WML foram estimadas de maneira análoga.

Para a construção do fator momento (WML), utilizou-se o retorno acumulado nos últimos 6 meses. Optou-se por essa janela pelo fato de Jagadeesh e Titman (1993, 1999) terem encontrado evidências de que as carteiras de momento construídas com base nos últimos 6 meses e mantidas pelos próximos 6 meses serem as que apresentaram maior retorno anormal. Bonomo e Agnol (2003) encontraram evidências de ganhos com estratégia contrária (venda de vencedoras e compra de perdedoras) no mercado acionário brasileiro no horizonte de 3 meses a 3 anos, mas não encontraram evidências de ganhos com estratégias de momento. O período analisado foi de 1986 a 2000. Optou-se nesse trabalho por manter a carteira autofinanciada comprada em ações vencedoras e vendida em ações perdedoras. Se os fundos brasileiros estiverem explorando estratégias de momento, devem apresentar sensibilidade positiva a esse fator. Se estiverem explorando estratégias contrárias, devem apresentar sensibilidade negativa. Se não estiverem explorando nenhuma estratégia, a sensibilidade deve ser nula. A Tabela 8 contém as estatísticas descritivas dos três fatores. 
Tabela 8

Estatística descritiva dos fatores SMB, HML e WML

\begin{tabular}{l|c|c|c|c}
\hline \multicolumn{1}{c|}{ Variável } & Média & Máximo & Mínimo & Desvio Padrão \\
\hline PL/Preço & $-0,0361$ & 0,2059 & $-0,3981$ & 0,0843 \\
Size & 0,0045 & 0,2562 & $-0,1876$ & 0,0797 \\
Momentum & $-0,0139$ & 0,1731 & $-0,2192$ & 0,0687 \\
\hline \multicolumn{2}{l}{ Fonte: Análise }
\end{tabular}

Adotou-se como fator de market timing o quadrado do retorno em excesso da carteira de mercado, conforme proposto por Treynor e Mazuy (1966).

Para cada um dos fundos, foi feita uma regressão múltipla por mínimos quadrados corrigidas pelo procedimento de Newey-West de acordo com a equação (2). O procedimento de Newey-West se justifica porque, de acordo com o teste de White, em $93,1 \%$ da amostra de fundos considerando retornos líquidos rejeitou-se a hipótese de homoscedasticidade, e de acordo com o teste de Durbin-Watson, em 31,4\% rejeitou-se a hipótese de ausência de correlação serial de primeira ordem. Explicações sobre os testes de White, Durbin-Watson e a tabela de valores críticos de Durbin-Watson podem ser obtidas em Greene (2000).

$$
\begin{aligned}
\left(R_{c}-R_{f}\right) & =\alpha+\beta_{1}\left(R m_{i}-R_{f}\right)+\beta_{2} S M B+\beta_{3} H M L \\
& +\beta_{4} W M L+\beta_{5}\left(R m_{i}-R_{f}\right)^{2}+\epsilon
\end{aligned}
$$

A equação (2) permite analisar o sinal e a significância da constante $\alpha$, que mede a habilidade de seletividade. Tem-se que $R c-R f$ é o excesso de retorno do fundo; $R m_{i}-R_{f}$ é o excesso de retorno do índice $i$, que pode ser IBOVESPA, IBX ou IBA de acordo com o benchmark do fundo; SMB é o retorno do fator tamanho, ou seja, retorno da carteira das menores empresas menos o retorno da carteira das maiores empresas; HML é o retorno do fator razão entre valor contábil e valor de mercado do patrimônio líquido; WML é o retorno do fator momento, medido como sendo o retorno das ações ganhadoras menos o retorno das ações perdedoras; $\left(R m_{i}-R_{f}\right)^{2}$ é o fator de market timing proposto por Treynor e Mazuy (1966) e $\epsilon$ é o erro da regressão.

Foram estimadas 626 regressões considerando o retorno líquido dos fundos, É importante ressaltar que as regressões foram feitas separadamente para cada fundo de acordo com a disponibilidade dos dados de cada um, ou seja, nos casos dos fundos que não apresentaram informações para todo o período (casos de novos fundos ou de fundos que deixaram de existir), a estimação só considerou o intervalo para o qual ele apresentou retornos.

Em seguida, foram estimadas outras 507 regressões de acordo com a equação (2), considerando as rentabilidades brutas. Essa prática permite analisar o impacto da taxa de administração na rentabilidade que chega ao investidor. Esses retornos brutos foram estimados de acordo com a equação (3).

$$
R_{\text {bruto }}=R_{\text {líquido }}+\left((1+\text { taxa de administração })^{1 / 12}-1\right)
$$




\section{Resultados}

A Tabela 9 apresenta os resultados de significância e sinal da constante alfa para os 626 fundos analisados de acordo com o retorno líquido. Considerandose um nível de significância de 5\%, os resultados mostram que apenas 30 (4,8\%) fundos apresentaram alfa significantemente positivo, sendo praticamente todos de gestão ativa (29) e indexados ao IBOVESPA (28). Apenas 1 dos fundos ativos indexados ao IBX apresentou alfa positivo, e nenhum dos fundos indexados ao IBA apresentou evidência de habilidade superior. Percebe-se ainda que o número de fundos com alfa significantemente negativo é maior que o número de fundos significativamente positivos, $1,5 \%$, sendo a grande maioria de fundos ativos.

Tabela 9

Análise da significância e sinal da constante alfa

\begin{tabular}{l|cc|cc}
\hline \multirow{2}{*}{ Fundos } & \multicolumn{2}{|c|}{ Alfas Positivos } & \multicolumn{2}{c}{ Alfas Negativos } \\
\cline { 2 - 5 } & Quantidade & $\%$ & Quantidade & $\%$ \\
\hline Total de Fundos & 30 & $4,8 \%$ & 72 & $11,5 \%$ \\
Ativos & 29 & $4,6 \%$ & 65 & $10,4 \%$ \\
Passivos & 1 & $0,2 \%$ & 7 & $1,1 \%$ \\
\hline Ativos IBOVESPA & 28 & $4,5 \%$ & 46 & $7,3 \%$ \\
Passivos IBOVESPA & 1 & $0,2 \%$ & 5 & $0,8 \%$ \\
Ativos IBX & 1 & $0,2 \%$ & 13 & $2,1 \%$ \\
Passivos IBX & 0 & $0,0 \%$ & 2 & $0,3 \%$ \\
Ativos IBA & 0 & $0,0 \%$ & 6 & $1,0 \%$ \\
Passivos IBA & 0 & $0,0 \%$ & 0 & $0,0 \%$ \\
\hline \multicolumn{2}{l|}{ Fonte: Análise dos autores, com base nos dados do Sistema de }
\end{tabular}

Informação (SI-ANBID) versão 4.1. e Economática.

A evidência encontrada está em linha com as conclusões de Jensen (1968) e Carhart (1997), a qual diz que poucos são os fundos ativos que conseguem gerar retornos superiores para os investidores, e que, pelo contrário, existem mais fundos ativos com desempenho inferior ao equilíbrio de mercado. Isso pode ter ocorrido em função das elevadas despesas dos fundos. Conforme indicam as tabelas 6 e 7, $27 \%$ dos fundos cobram taxas de administração superior a $3 \%$, sendo que a média das taxas de administração cobradas dos fundos ativos é bem superior à dos fundos passivos.

A tabela 10 contém estatísticas descritivas da amostra de todos os 626 alfas estimados pelas 626 regressões. Os alfas dos fundos passivos e dos fundos ativos são em média significativamente menores que zero a um nível de 5\%. Não se rejeita a hipótese de que, em média, os fundos ativos tiveram o mesmo desempenho medido pelo alfa que os fundos passivos. 
Tabela 10

Estatísticas descritivas da amostra de alfas estimados

\begin{tabular}{l|c|c|c|c|c|c}
\hline & $\begin{array}{c}\text { Alfa } \\
\text { Médio }\end{array}$ & $\begin{array}{c}\text { Alfa } \\
\text { Máximo }\end{array}$ & $\begin{array}{c}\text { Alfa } \\
\text { Mínimo }\end{array}$ & $\begin{array}{c}\text { Desvio } \\
\text { Padrão }\end{array}$ & $\begin{array}{c}\text { Estatística } \\
T\end{array}$ & $n$ \\
\hline Todos os Fundos & $-0,001319$ & 0,133306 & $-0,087982$ & 0,014877 & $-2,217473$ & 626 \\
Fundos Ativos & $-0,001323$ & 0,133306 & $-0,087982$ & 0,015472 & $-2,054228$ & 577 \\
Fundos Passivos & $-0,001264$ & 0,008411 & $-0,008513$ & 0,003038 & $-2,912182$ & 49 \\
\hline
\end{tabular}

Fonte: Análise dos autores, com base nos dados do Sistema de Informação (SI-ANBID)

versão 4.1. e Economática.

A Tabela 11 apresenta a quantidade de betas significativos a 5\% para cada um dos fatores de características do modelo de Carhart (1997): tamanho (SMB), razão entre valor contábil e valor de mercado do patrimônio líquido (HML) e momento (WML) e para o fator de market timing. Como pode ser observado, 13,7\% da amostra de fundos ativos explorou o efeito tamanho, 9,7\% explorou o efeito razão entre valor contábil e valor de mercado do patrimônio líquido, $10,5 \%$ explorou o efeito momento e $18,7 \%$ explorou market timing. A maior parte dos fundos apresenta sinal negativo para market timing, indicando que seus administradores ou aumentaram o beta do fundo quando os retornos do índice caíram, ou diminuíram o beta do fundo quando os retornos do índice aumentaram, ou seja, o contrário do que seria recomendado. Alguns fundos passivos aparentemente exploraram características: $4,1 \%$ explorou a razão entre valor contábil e valor de mercado do patrimônio líquido, $6,1 \%$ o efeito momento e $20,4 \%$ o market timing.

Quando se observa a sensibilidade de fundos ao fator momento, percebe-se que 28 dos 626 fundos apresentam sensibilidade significativamente positiva e 38 negativa. Esse resultado indica que um percentual razoável de fundos ativos explora o diferencial de retornos entre ações ganhadoras e perdedoras, mas o maior número destes fundos explora a estratégia contrária, o que seria esperado por Bonomo e Agnol (2003). Por fugir ao escopo dessa pesquisa, não foi analisado se a estratégia de momento ou estratégia contrária trouxe ganhos anormais nesse período.

Tabela 11

Número de fundos com coeficiente significativo a 5\% aos fatores de característica de Cahart e market timing

\begin{tabular}{c|c|c|c|c}
\hline Fatores & Sinal & $\begin{array}{c}\text { Total dos } \\
\text { Fundos }\end{array}$ & $\begin{array}{c}\text { Fundos } \\
\text { Ativos }\end{array}$ & $\begin{array}{c}\text { Fundos } \\
\text { Passivos }\end{array}$ \\
\hline SMB & positivo & 82 & 82 & 0 \\
& negativo & 4 & 4 & 0 \\
& total & $\mathbf{8 6}$ & $\mathbf{8 6}$ & $\mathbf{0}$ \\
\hline HML & positivo & 18 & 18 & 0 \\
& negativo & 43 & 41 & 2 \\
& total & $\mathbf{6 1}$ & $\mathbf{5 9}$ & $\mathbf{2}$ \\
\hline WML & positivo & 28 & 25 & 3 \\
& negativo & 38 & 38 & 0 \\
& total & $\mathbf{6 6}$ & $\mathbf{6 3}$ & $\mathbf{3}$ \\
\hline Market timing & positivo & 43 & 39 & 4 \\
& negativo & 74 & 68 & 6 \\
& total & $\mathbf{1 1 7}$ & $\mathbf{1 0 7}$ & $\mathbf{1 0}$ \\
\hline Fonte: Análise dos autores, com base nos dados do Sistema de
\end{tabular}

Informação (SI-ANBID) versão 4.1. e Economática. 
Com o intuito de observar a relação entre o tamanho de um fundo com seu desempenho, foi feita uma regressão entre os 626 alfas estimados e o logarítimo do patrimônio líquido de cada um dos fundos. Constatou-se que o coeficiente da regressão apresentou uma estatística t igual a 4,48, e portanto uma relação positiva e significativa a 5\%. Isso significa que quanto maior o tamanho do fundo, melhor o desempenho. Bodie et alii (2008, p.369) argumentam que se os preços dos ativos forem próximos a seus valores justos, apenas administradores de grandes fundos serão capazes de gerar ganhos suficientes para que a exploração de pequenas ineficiências valha a pena. Chamam esse aspecto da discussão de eficiência de mercado de magnitude. Uma interpretação alternativa é que embora gestores de fundos menores possam ter tido habilidade superior e gerado ganhos anormais, esses não foram grandes o suficiente para pagar os custos da análise fundamentalista, e resultaram em alfas negativos para o investidor final. Grinblatt e Titman (1989), quando analisaram retornos brutos, encontraram evidências de que o desempenho superior existe particularmente em fundos de crescimento e fundos de menor patrimônio líquido. Mas como esses fundos têm maiores despesas (corretagem, taxa de administração e outros custos de transação), a análise feita com retornos líquidos não apresenta desempenho superior.

A Tabela 12 apresenta uma análise da significância dos alfas estimados com retornos líquidos quando se excluem os fundos com patrimônio líquido inferior a R \$ 1,5 milhões. Percebe-se que a um nível de significância de 5\%, o número de fundos com Alfa positivo e significativo continuou praticamente o mesmo, com uma redução de apenas 3 fundos, indicando que os fundos que apresentaram habilidade de seletividade superior foram os fundos maiores. Também observa-se que o número de fundos com alfa negativo e significativo caiu de 72 para 47 , ou seja, 25 dos fundos com alfa negativo tinham patrimônio líquido inferior a $\mathrm{R}$ \$ 1,5 milhões. Isso reforça idéia de que fundos menores geram os piores desempenhos quando considerados retornos líquidos.

Tabela 12

Análise da significância e sinal da constante alfa quando eliminados os fundos com patrimônio líquido inferior a $\mathrm{R} \$ 1,5$ milhões

\begin{tabular}{c|c|c}
\hline Alfas & $\begin{array}{c}\text { Amostra com } \\
\text { PL mínimo de } \\
\mathrm{R} \$ 1,5 \text { milhões }\end{array}$ & $\begin{array}{c}\text { Amostra } \\
\text { completa }\end{array}$ \\
\hline positivos e significativos & 27 & 30 \\
negativos e significativos & 47 & 72 \\
\hline \multicolumn{2}{l|}{ Fonte: Análise dos autores, com base nos dados do Sistema } \\
de Informação (SI-ANBID) versão 4.1. e Economática.
\end{tabular}

As regressões de acordo com a equação (2) foram repetidas para os 507 fundos em que foi possível estimar séries de retornos brutos. Essa análise permite ter uma idéia se as taxas de administração estão sendo responsáveis pela eliminação de ganhos anormais, conforme verificado por Grinblatt e Titman (1989). É necessário reconhecer que os resultados são apenas indicativos, pois existem várias limitações na análise. Não foram incluídas taxas de desempenho, custos de transação e a taxa 
de administração foi considerada fixa e igual à divulgada pela ANBID, CVM e Quantum para a última observação do fundo, embora na prática possivelmente a taxa tenha variado ao longo do tempo e tenha sido diferente da divulgada. A análise da significância e sinal dos alfas encontra-se na Tabela 13 e estatísticas descritivas dos alfas estimados na Tabela 14.

Tabela 13

Número de fundos com Alfas significativos quando estimados com rentabilidades brutas

\begin{tabular}{l|cc|cc}
\hline \multirow{2}{*}{ Fundos } & \multicolumn{2}{|c|}{ Alfas Positivos } & \multicolumn{2}{c}{ Alfas Negativos } \\
\cline { 2 - 5 } & Quantidade & $\%$ & Quantidade & $\%$ \\
\hline Total de Fundos & 52 & $10,3 \%$ & 34 & $6,7 \%$ \\
Ativos & 50 & $9,9 \%$ & 33 & $6,5 \%$ \\
Passivos & 2 & $0,4 \%$ & 1 & $0,2 \%$ \\
\hline Ativos IBOVESPA & 46 & $9,1 \%$ & 25 & $4,9 \%$ \\
Passivos IBOVESPA & 2 & $0,4 \%$ & 0 & $0,0 \%$ \\
Ativos IBX & 4 & $0,8 \%$ & 8 & $1,6 \%$ \\
Passivos IBX & 0 & $0,0 \%$ & 1 & $0,2 \%$ \\
Ativos IBA & 0 & $0,0 \%$ & 0 & $0,0 \%$ \\
Passivos IBA & 0 & $0,0 \%$ & 0 & $0,0 \%$ \\
\hline \multicolumn{2}{r}{ Fonte: Análise dos autores, com base nos dados do Sistema de Informação }
\end{tabular}

(SI-ANBID) versão 4.1. e Economática.

Tabela 14

Estatísticas descritivas dos alfas estimados com rentabilidades brutas

\begin{tabular}{l|c|c|c|c|c|c}
\hline & Alfa & Alfa & Alfa & Desvio & Estatística & $n$ \\
& Médio & Máximo & Mínimo & Padrão & $T$ & \\
\hline Todos os Fundos & 0,001071 & 0,133973 & $-0,084237$ & 0,013465 & 1,791746 & 507 \\
Fundos Ativos & 0,001178 & 0,133973 & $-0,084237$ & 0,014115 & 1,788075 & 459 \\
Fundos Passivos & 0,000052 & 0,010077 & $-0,008259$ & 0,003025 & 0,118933 & 48 \\
\hline
\end{tabular}
versão 4.1. e Economática.

Os alfas dos fundos ativos são em média significativamente maior que zero a um nível de $10 \%$. O número de fundos com alfa positivo e significativo mais que dobrou, e o número de fundos com alfa significativamente negativo caiu de $11,5 \%$ para $6,7 \%$ da amostra. Considerando-se que não foram incluídas taxas de desempenho e custos de corretagem, que provavelmente aumentariam mais o número de fundos com habilidade de seletividade significativa, pode-se interpretar que os resultados com retornos brutos estão de acordo com as evidências empíricas encontradas por Grinblatt e Titman (1989) e que a taxa de administração é um importante fator na determinação da rentabilidade de um fundo.

A Tabela 15 apresenta os resultados de uma análise dos alfas estimados com retornos brutos quando se elimina da amostra os fundos com patrimônio líquido menor que $\mathrm{R} \$ 1,5$ milhão. Pode-se perceber que o número de fundos com alfa positivo e significativo diminuiu de 52 para 47, indicando que 5 fundos com patrimônio líquido menor que R \$ 1,5 milhões tiveram habilidade de seletividade significativa, quando se consideram retornos brutos. Esse número é um pouco superior à mesma análise feita com retornos líquidos, mas ainda mostra uma grande concentração das evidências de habilidade superior em fundos grandes. Por outro lado, o número 
de fundos com alfa negativo e significativo caiu de 34 para 25 fundos, indicando que 9 fundos que tiveram desempenho pior que o equilíbrio de mercado tinham patrimônio líquido inferior a $\mathrm{R}$ \$ 1,5 milhões (na análise de retornos líquidos, o número de fundos pequenos com desempenho ruim foi 25). Houve portanto uma menor concentração de fundos pequenos com habilidade negativa, o que indica que o fato dos menores fundos gerarem retornos anormais negativos para o investidor não advém necessariamente de habilidade ruim, mas do fato da magnitude desses retornos anormais não ser suficientemente grandes para compensar as taxas de administração necessárias para pagar os custos da análise fundamentalista.

Embora a análise com retornos brutos indique uma concentração menor dos piores resultados em fundos menores, quando se faz uma regressão do alfa em relação ao logaritmo do patrimônio líquido observa-se um coeficiente positivo com estatística t igual a 3,89. Embora menos significativo que os resultados líquidos, o resultado mostra que a relação positiva entre alfa e tamanho do fundo ainda é bastante significativa. A inclusão de taxa de desempenho e corretagem talvez pudesse fornecer indícios de que realmente o pior desempenho dos fundos menores não é fruto necessariamente de uma habilidade pior, mas da menor diluição dos custos.

\section{Tabela 15}

Análise da significância e sinal da constante alfa estimada com retornos brutos quando eliminados os fundos com patrimônio líquido inferior a $\mathrm{R} \$ 1,5$ milhões

\begin{tabular}{|c|c|c|}
\hline Alfas & $\begin{array}{l}\text { Amostra com } \\
\text { PL mínimo de } \\
\text { R } \$ 1,5 \text { milhões }\end{array}$ & $\begin{array}{l}\text { Amostra } \\
\text { completa }\end{array}$ \\
\hline positivos e significativos & 47 & 52 \\
\hline negativos e significativos & 25 & 34 \\
\hline
\end{tabular}

\section{Conclusão}

Os resultados obtidos mostram que um número razoável de fundos ativos brasileiros explorou anomalias conhecidas, como tamanho (13,42\% da amostra), razão entre valor contábil e valor de mercado de patrimônio líquido $(8,79 \%)$ e momento $(10,54 \%)$.

Quando as regressões foram feitas com estimativas de retornos líquidos, apenas $4,6 \%$ dos fundos, todos ativos, foram capazes de ter retornos significativos superiores ao desempenho de equilíbrio de mercado, e 14,4\% dos fundos tiveram desempenho sigificativamente inferior ao mercado. Não se rejeita a hipótese também que o desempenho dos fundos ativos é igual ao dos fundos passivos.

$\mathrm{O}$ resultado com retornos brutos eleva o percentual de fundos com habilidade de seletividade para $10 \%$ da amostra, e reduz o percentual de fundos com desempenho pior que o mercado para $5,3 \%$. Os fundos ativos tiveram em média desempenho positivo e significativo. 
Os resultados estão de acordo com as evidências encontradas em Grinblatt e Titman (1989) e Carhart (1997) nos Estados Unidos, pois apenas um pequeno grupo de administradores excepcionais pode consistentemente superar o mercado. Também estão de acordo com a versão da hipótese de eficiência de mercado de Jensen (1978), segundo a qual os preços refletem as informações disponíveis até o ponto em que o benefício marginal de utilizar a informação (lucros a serem realizados) não excede os custos marginais, ou seja, os ganhos anormais provenientes da exploração de anomalias são, em média, suficientes apenas para pagar as despesas da gestão ativa.

Rochman e Eid Junior (2006) encontraram evidências de que os fundos ativos de ações e multimercado no Brasil criam valor para o acionista. Este resultado diverge das evidências encontradas nesse trabalho. Possíveis explicações são diferenças no período, tamanho da amostra e modelo de equilíbrio utilizado. Rochman e Eid Junior (2006) analisaram 129 fundos ativos e 34 passivos no período de Janeiro de 2001 a Janeiro de 2006. Utilizaram como modelo de equilíbrio o sugerido por Jensen (1968), que contém um único fator sistemático: o retorno em excesso do índice de mercado. Neste artigo foi analisada uma amostra bem maior de fundos de ações (577 fundos ativos e 49 passivos), num período mais amplo (janeiro de 1996 a outubro de 2006) e com um modelo multifatorial que controla por estratégias geralmente utilizadas por fundos de ações. Também foram encontradas nesse trabalho evidências de que apenas uma pequena parcela de fundos é bem sucedida em explorar a estratégia de market timing. Esse resultado está de acordo com os de Leusin e Brito (2006) e Franz e Figueiredo (2003).

A análise com retornos brutos apresenta limitações, pois foram utilizadas as taxas de administração declaradas no último mês em que se observou a cota do fundo, mas que pode ter divergido da taxa real descontada do investidor. Também não foram considerados taxas de desempenho e outros custos de transação. Portanto, pode-se concluir que se a taxa de administração efetivamente cobrada for igual à declarada, cerca de $10 \%$ dos gestores de fundos ativos tem ganhos anormais brutos significativos, indicando haver o fenômeno de hot hands no mercado brasileiro, fenômeno também encontrado no mercado americano de acordo com Hendricks et alii (1993).

Os resultados desse exercício são interessantes, e estão de acordo com a evidência de que os maiores fundos geram os maiores ganhos anormais, e que os menores fundos tiveram desempenho negativo. Bodie et alii (2008) discutem que apenas grandes fundos são capazes de gerar ganhos suficientemente grandes para pagar os custos com a análise fundamentalista, devido ao aspecto magnitude. Embora a maior parte dos fundos gere desempenho abaixo do equilíbrio de mercado para os investidores, isso é decorrente de custos de transações que extrapolam os ganhos com anomalias, e não por falta de habilidade de seletividade. Carhart (1997) reforça essa idéia ao afirmar que fundos com elevadas taxas de administração tem suas rentabilidades líquidas prejudicadas.

Por englobar todos os fundos abertos de ações ativos e passivos, pode-se dizer 
que a amostra adotada é representativa de mercado e que, portanto, os resultados encontrados podem ser estendidos para o mercado Brasileiro como um todo. Uma possível extensão desse trabalho seria coletar as informações trimestrais das carteiras de fundos de ações divulgadas à CVM e a partir dessas estimar custos de corretagem e séries de retornos brutos mais precisas, conforme foi feito por Grinblatt e Titman (1989). Isso possibilitaria investigar mais profundamente se a concentração dos piores resultados nos fundos menores e dos melhores resultados em fundos maiores persistiria e se os menores fundos tem habilidade superior.

\section{Referências}

Andaku, F. T. A. \& Pinto, A. C. F. (2003). Persistência de desempenho de fundos de investimento em ações no Brasil. Revista de Economia e Administração, 2(2):23-33.

Bodie, Z., Kane, A., \& Marcus, A. J. (2008). Investments. McGraw-Hill, Boston, MA, 7 edition.

Bonomo, M. \& Agnol, I. D. (2003). Retornos anormais e estratégias contrárias. Revista Brasileira de Finanças, 1(2):165-215.

Carhart, M. M. (1997). On persistence in mutual fund performance. Journal of Finance, 52(1):57-82.

Fama, E. (1972). Components of investment performance. Journal of Finance, 27(3):551-567.

Fama, E. \& French, K. R. (1993). Common risk factors in the returns on stocks and bonds. Journal of Financial Economics, 33(1):3-56.

Franz, P. R. G. \& Figueiredo, A. C. (2003). Avaliação da capacidade de market timing dos administradores de fundos mútuos de ações no Brasil. Revista de Economia e Administração, 2(1):33-46.

Greene, W. H. (2000). Econometric Analysis. Prentice Hall, Upper Saddle River, NJ, 4 edition.

Grinblatt, M. \& Titman, S. (1989). Mutual fund performance: An analysis of quarterly portfolio holdings. Journal of Business, 62(3):393-416.

Hendricks, D., Patel, J., \& Zechauser, R. (1993). Hot hands in mutual funds: Short-run persistence of relative performance, 1974 - 1988. Journal of Finance, 48(1):93-130.

Henriksson, R. D. (1984). Market timing and mutual fund performance: An empirical investigation. Journal of Business, 57(1):73-96. 
Henriksson, R. D. \& Merton, R. C. (1981). On market timing and investment performance. II. statistical procedures for evaluating forecasting skills. The Journal of Business, 54(4):513-533.

Jagadeesh, N. \& Titman, S. (1993). Returns to buying winners and selling losers: Implication for stock market efficiency. Journal of Finance, 48(1):65-91.

Jagadeesh, N. \& Titman, S. (1999). Profitability of momentum strategies: An evaluation of alternative explanation. Working paper 7159. National Bureau of Economics Research.

Jensen, M. (1968). The performance of mutual funds in the period 1945-1964. Journal of Finance, 23(2):389-416.

Jensen, M. (1978). Some anomalous evidence regarding market efficiency. Journal of Financial Economics, 6:95-101.

Lee, C.-F. \& Rahman, S. (1990). Market timing, selectivity, and mutual fund performance: An empirical investigation. Journal of Business, 63(2):261-278.

Leusin, L. \& Brito, R. D. (2006). Market timing e avaliação de desempenho dos fundos brasileiros. VI Encontro Brasileiro de Finanças. Vitória, ES.

Monteiro, R. C. (2007). Persistência de performance nos fundos de investimentos em ações no Brasil. Dissertação de Mestrado. VII Encontro Brasileiro de Finanças. São Paulo, SP.

Rochman, R. R. \& Eid Junior, W. (2006). Fundos de investimento ativos e passivos no Brasil: Comparando e determinando os seus desempenhos. EnANPAD 2006 - Salvador/BA.

Treynor, J. L. \& Mazuy, K. (1966). Can mutual funds outguess the market? Harvard Business Review, 43:131-136. 